\title{
Doxorubicin metabolism moderately attributes to putative toxicity in prodigiosin/doxorubicin synergism in vitro cells
}

\author{
Shian-Ren Lin ${ }^{1} \cdot$ Chun-Shu Lin ${ }^{2} \cdot$ Ching-Cheng Chen ${ }^{3} \cdot$ Feng-Jen Tseng ${ }^{4} \cdot$ Tsung-Jui Wu $^{4} \cdot$ Lebin Weng $^{5}$. \\ Ching-Feng Weng ${ }^{5,6}$ (1)
}

Received: 5 March 2020 / Accepted: 28 July 2020 / Published online: 4 August 2020

(c) The Author(s) 2020

\begin{abstract}
Doxorubicin (Dox) is a widely neoplasm chemotherapeutic drug with high incidences of cardiotoxicity. Prodigiosin (PG), a red bacterial pigment from Serratia marcescens, has been demonstrated to potentiate Dox's cytotoxicity against oral squamous cell carcinoma cells through elevating Dox influx and identified as a Dox enhancer via PG-induced autophagy; however, toxicity of normal cell remains unclear. This study is conducted to evaluate putative cytotoxicity features of PG/ Dox synergism in the liver, kidney, and heart cells and further elucidate whether PG augmented Dox's effect via modulating Dox metabolism in normal cells. Murine hepatocytes FL83B, cardio-myoblast h9c2, and human kidney epithelial cells HK-2 were sequentially treated with PG and Dox by measuring cell viability, cell death characteristics, oxidative stress, Dox flux, and Dox metabolism. PG could slightly significant increase Dox cytotoxicity in all tested normal cells whose toxic alteration was less than that of oral squamous carcinoma cells. The augmentation of Dox cytotoxicity might be attributed to the increase of Dox-mediated ROS accumulation that might cause slight reduction of Dox influx and reduction of Dox metabolism. It was noteworthy to notice that sustained cytotoxicity appeared in normal cells after PG and Dox were removed. Taken together, moderately metabolic reduction of Dox might be ascribed to the mechanism of increase Dox cytotoxicity in PG-induced normal cells; nevertheless, the determination of PG/Dox dose with sustained cytotoxicity in normal cells needs to be comprehensively considered.
\end{abstract}

Keywords Doxorubicin $\cdot$ Prodigiosin $\cdot$ Drug synergism $\cdot$ Drug metabolism $\cdot$ Cardiotoxicity

Ching-Feng Weng

cfweng-cfweng@hotmail.com

1 Graduate Institute of Cancer Biology and Drug Discovery, Taipei Medical University, Taipei 110, Taiwan

2 Department of Radiation Oncology, Tri-Service General Hospital, National Defense Medical Center, Taipei, Taiwan

3 Camillian Saint Mary's Hospital Luodong, Luodong 26546, Yilan, Taiwan

4 Department of Orthopedics, Hualien Armed Force General Hospital, Hualien 97144, Taiwan

5 Functional Physiology Section, Department of Basic Medical Science, Xiamen Medical College, Xiamen 361023, China

6 Institute of Respiratory Disease, Department of Basic Medical Science, Xiamen Medical College, Xiamen 361023, China

\section{Introduction}

Rendering non-tissue specific characteristics, doxorubicin (Dox) has wide indications, e.g., leukemia, neuroblastoma, breast, and ovarian carcinoma, and most recurrent or metastatic cancer in cancer regimen [1], and it also has severe adverse effects in cancer patients. $31-51 \%$ of Dox is excreted via biliary tract in their original form and $<10 \%$ of Dox metabolite is eliminated via urine after intake [2, 3]. Three pathways of Dox metabolism are under investigation: one-electron reduction, two-electron reduction, and deglycosylation, which carried about $50 \%$ of Dox out of cells (remaining $50 \%$ will be excreted from their original form) [4]. One-electron reduction occurs in mitochondria which operates by NADH-ubiquinone oxidoreductase (NDUFS), xanthine oxidase (XOD), and a cytosolic enzyme NADPH: quinone oxidoreductase (NQO). The end-product of oneelectron reduction is Dox-semiquinone, whose ROS activity is the main cause of oxidative stress. Two-electron reduction 
pathways which aldo/keto reductase (AKR) and carbonyl reductase (CBR) are the main metabolic pathway of Dox detoxification. Dox-deglycosylation is a minor route, which produces Dox-aglycone by NQO [5-7]. Along with the trigger mechanism, the cytotoxicity of Dox is considered to be via two approaches: apoptotic activation, which refers to hematopoietic inhibition, mucositis, and enteritis; and oxidative stress which causes most hepatotoxicity, nephrotoxicity, and cardiotoxicity $[8,9]$.

In clinic, Dox-induced cardiotoxicity is the most divergent of concerned toxicity. The incidence of Dox-induced cardiomyopathy is positively correlated to cumulative dose, which increased from $4 \%\left(500-550 \mathrm{mg} / \mathrm{m}^{2}\right)$ to $36 \%$ $\left(>600 \mathrm{mg} / \mathrm{m}^{2}\right)$ [10]. To minimize the adverse effect of Dox and keeping its anticancer efficacy, nanocarriers have been developed and some researchers have focused on dissolving these obstacles via liposomal-coated Dox (Lipo-Dox). However, the cytotoxicity of liposomal Dox is 10 times less than that of free-form Dox, which might increase the risk of chemoresistance induced [11]. The lower cytotoxicity of Lipo-Dox than free form of Dox sheds light on the possibility of adjuvant involvement in promoting Dox's antitumor efficacy without raising its normal cell toxicity.

In cancer treatment, the term "adjuvant therapy" is referred to the regimen based on the synergistic effect or combination of two or more therapeutic drugs, usually a lower-dose therapy followed by a primary therapy for reducing cancer recurrence [12]. Clinically, adjuvant therapies for cancer treatment are usually combining surgery with chemotherapy or radiotherapy for efficaciously potentiating the effects of two treatments or chemicals, and whether this efficacy is indicative of a positive or negative response [13]. Generally, it is common to combine two current chemotherapeutic agents in one regimen, i.e., Dox with tamoxifen, paclitaxel plus Dox, and cyclophosphamide or carboplatin with docetaxel [14]. However, clinical trials revealed the effects of polymorphism for adjuvant chemotherapy and the biomarkers for efficacy estimation were not completely or totally defined [15-18]. Accordingly, additional agents for enhancing the efficacy of adjuvant chemotherapeutic drugs or extending patient polymorphism need to developed using this new strategy. Previously, Dox accumulation caused by prodigiosin (PG)-priming has been confirmed in OSCC through the elevation of Dox influx [19]. However, the caused damage of PG/Dox combination in known organs, e.g., liver, kidney, and heart were not assessed. In this study, putative hepato-, nephro-, and cardiotoxicity of PG/Dox combinations and the underlying regulation are explored using an in vitro cellular assay.

\section{Material and methods}

\section{Chemicals}

Chemicals were obtained from various sources: purified PG was from Dr. Yu-Hsin Chen (National Museum of Marine Biology and Aquarium, Pingtung, Taiwan), liposome-coated Dox nanoparticle (Lipo-Dox) was provided by TTY Biopharm Company Limited (Taipei, Taiwan), general chemicals from Sigma-Aldrich (Merck KGaA, Darmstadt, Germany), medium and reagents for cell culture from Thermo-Fisher (Waltham, MA, USA), and inhibitors, antibiotics for selection, and lentiviral activation particle from Santa Cruz Biotechnology (Dallas, TX, USA) in this study. All reagents for cell culture were dissolved in dimethyl sulfoxide as a 1000X working stock and stored under dark, $-20^{\circ} \mathrm{C}$ environment except lentiviral activation particle $\left(\right.$ at $\left.-80^{\circ} \mathrm{C}\right)$ and antibiotics for selection $\left(4{ }^{\circ} \mathrm{C}\right)$.

\section{Cell culture}

A total of 3 cell lines were from different sources: murine normal hepatocyte FL83B and cardio-myoblast $h 9 \mathrm{c} 2$ from Bioresource Collection and Research Center (BCRC, Hsinchu, Taiwan), and human renal cortex/proximal tubule epithelial cell HK-2 from Prof. Yaw-Syan Fu (Department of Biomedical Science and Environment Biology, Kaohsiung Medical University, Kaohsiung, Taiwan) were applied to test the hypothesis in this study. Three cell lines were cultured in different mediums and were all supplied with $10 \%$ fetal bovine serum and $1 \%$ antibiotic/antimycotic and $0.2 \%$ normocin $^{\mathrm{TM}}$ : FL83B in Kaighn-modified Ham's F12 medium (F12K), h9c2 in Dulbecco's modified Eagle medium (DMEM), and HK-2 in DMEM/F12 with additional $0.5 \mathrm{ng} /$ mL epidermal growth factor (EGF). The cultural condition was set as $37{ }^{\circ} \mathrm{C}, 5 \% \mathrm{CO}_{2}$, and saturated humidity. Culture medium was renewed once every 2 days. As cell confluence reached $80 \%$, cells were detached by $0.25 \%$ Trypsin/EDTA for experiments. Cell passage was kept within 20 passages for controlling the uniformity of experiments.

\section{Cytotoxicity assay}

Cytotoxicity analysis was performed by MTT (3-(4,5-dimethylthiazol-2-yl)-2,5-diphenyltetrazolium bromide) staining with viable cells as described in a previous study [19]. Briefly, $1 \times 10^{4}$ cells/well of normal cells were inoculated into 96-well plate and incubated under cultural conditions overnight. Then, cells were treated with $0.5 \mu \mathrm{M}$ of PG for $12 \mathrm{~h}$ followed by $2.5 \mu \mathrm{M}$ of Dox treatment for $12 \mathrm{~h}$. Afterward, $1 \mathrm{mg} / \mathrm{mL}$ MTT solution (in PBS) was added to 
the wells (final concentration $0.5 \mathrm{mg} / \mathrm{mL}$ ) and incubated for $4 \mathrm{~h}$. Finally, cultural medium was replaced with $50 \mu \mathrm{L}$ of dimethyl sulfoxide and the optical density (OD) at $570 \mathrm{~nm}$ was measured by a Multiskan ${ }^{\mathrm{TM}} \mathrm{FC}$ microplate photometer (Thermo-Fisher). Cytotoxicity was calculated from the OD ratio between untreated (control as 1) and treated groups.

\section{Cell cycle analysis}

Cell cycle of treated cells was measured by propidium iodide (PI)-staining and flow cytometry according to the literature [19]. $1 \times 10^{6}$ cells/well of normal cells were seeded into 6-well plate. Cells were treated with PG/Dox as described in the previous section. After treatment, cells and supernatant from each well was collected individually by $0.25 \%$ trypsin/ EDTA, washed by pre-warm PBS, and fixed by ice-cold 70\% ethanol/PBS under $-20^{\circ} \mathrm{C}$ at least overnight. Fixed cells were washed out ethanol by PBS and stained with $20 \mu \mathrm{g} / \mathrm{mL}$ of propidium iodide coupling with $0.1 \%$ Triton X-100 and $0.2 \mathrm{mg} / \mathrm{mL}$ RNase A for $1 \mathrm{~h}$ at $37^{\circ} \mathrm{C}$. Fluorescent intensity from PI in each cell was determined by Cytomics ${ }^{\mathrm{TM}} \mathrm{FC} 500$ flow cytometer (Beckman-Coulter, Fullerton, CA, USA). $1 \times 10^{4}$ data from each well were collected and plotted histogram by flow cytometry software. The gating of each cell cycle state was performed according to the histogram from untreated controls.

\section{Dox influx assay}

A Dox influx assay was performed by the accumulation of Dox-transporter substrate rhodamine 123 (R123) in the literature [19]. $1 \times 10^{4}$ cells/well of normal cells were inoculated into a 96-well plate. Then, the cells were incubated with $0.5 \mu \mathrm{M}$ PG for $12 \mathrm{~h}$ and $2 \mu \mathrm{M}$ R 123 at cultural conditions for $1 \mathrm{~h}$. The cells were washed by pre-warm PBS and lysed by $0.1 \%$ triton X-100 for $10 \mathrm{~min}$ and fluorescent intensity at $485 / 538 \mathrm{~nm}$ was determined by Alphascreen ${ }^{\mathrm{TM}}$ microplate reader (Perkin-Elmer, Waltham, MA, USA). Dox influx was represented by fluorescent percentage from untreated (control) and treated groups.

\section{Gene overexpression}

Lentiviral-mediated gene overexpression was performed by Lentiviral activation particle, which followed the protocol provided by the manufacturer (https://datasheets .scbt.com/protocols/CRISPR_Lenti_Activation_Protocol. pdf). $1.5 \times 10^{5}$ cells/well of each cell were inoculated into 12-well plate and incubated in culture conditions overnight for cell attachment. $10 \mu \mathrm{L}$ of lentivirus particle and 1.5 $\mu \mathrm{L}$ of $10 \mathrm{mg} / \mathrm{mL}$ polybrene were mixed in $1 \mathrm{~mL}$ of culture medium (with $2 \%$ FBS), added to each cell, and incubated in culture conditions overnight. After lentiviral infection, transfected cells were selected by incubation with $350 \mu \mathrm{g} /$ $\mathrm{mL}$ hygromycin $\mathrm{S}$ for 8-24 h. Transfection was re-confirmed by measurement of cytotoxicity after treating with selected antibiotics for $24 \mathrm{~h}$. Transfected cells were used to measure cytotoxicity change of PG/Dox compared with un-transfected cells and desired protein expression using Western blotting.

\section{Statistical analysis}

Collected data were plotted with GraphPad Prism V7.04 (La Jolla, CA, USA) and performed analysis of variance (ANOVA) coupled with Dunnett's test or Tukey's range test as Post hoc comparison. Data were shown by mean \pm SD from at least 3 independent experiments. As data were significantly different $(p<0.05)$ from untreated control or Dox alone, column of the data would be marked “*” or "\#”, respectively.

\section{Results}

\section{Cytotoxicity of PG/Dox synergism against normal cells}

To access putative toxicity from PG/Dox synergism in liver, kidney, and heart, FL83B (murine normal hepatocyte), HK-2 (human kidney proximal tubule epithelial cell), and h9c2 (murine normal cardio-myoblast) were sequentially treated with PG and Dox for $12 \mathrm{~h}$, respectively. Comparing with PG or Dox alone treatment for $12 \mathrm{~h}$, the cytotoxicity of PG/Dox exhibited significantly increase in $62.9 \pm 1.8 \%$ (FL83B), $40.8 \pm 0.8 \%$ (HK-2), and $74.5 \pm 0.7 \%$ (h9c2), respectively, whose cell viabilities after Dox treatment were $68.1 \pm 1.1 \%$ (FL83B), $47.7 \pm 1.7 \%$ (HK-2), and $74.6 \pm 0.5 \%$ (h9c2), respectively (Fig. 1). This result indicated cytotoxic enhancement also appeared in normal cells. However, the cell viability of $\mathrm{PG} /$ Dox in normal cells was higher trend (no significance) when compared with $2.5 \mu \mathrm{M}$ Dox treating for $24 \mathrm{~h}$. When the concentration of Dox alone increased to $5 \mu \mathrm{M}$, cell viability of three different cells dropped to $59.9 \pm 0.9 \%$ (FL83B), $30.3 \pm 1.2 \%$ (HK-2), and $53.4 \pm 0.7 \%$ (h9c2), respectively. There were all significantly lower than that of PG/Dox treatment (Fig. 1). The results indicated the enhancement of Dox cytotoxicity combined with PGpriming; however, its cytotoxic enhancement was not higher than that of Dox alone treated with $24 \mathrm{~h}$. Moreover, the range of cytotoxic enhancement in normal cells was smaller than OSCC [19], which could reinforce the potential of PG/Dox combination for clinical use. 


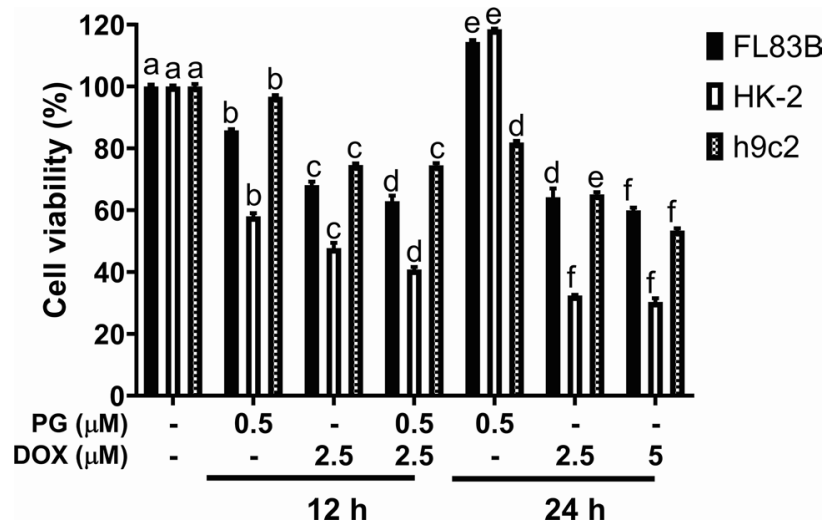

Fig. 1 Cytotoxicity of PG/Dox against normal cells. FL83B, HK-2, and h9c2 cells were sequentially treated with PG/Dox for 12 and 24 $\mathrm{h}$, respectively, and cell viability was measured by MTT assay. Data were represented with mean \pm SD from four independent experiments. Columns significantly different letter $(p<0.05)$ to each other would be labeled with different letters



Fig. 2 Cytotoxic alteration of PG/Dox by addition of autophagic inhibitors in normal cells. FL83B, HK-2, and h9c2 cells were treated with PG coupled with 3-methyladenine (3MA) or bafilomycin A1 (BA1) for $12 \mathrm{~h}$ following by Dox for $12 \mathrm{~h}$, respectively. Results were normalized with untreated control and shown in mean \pm SD from four independent experiments. "*" and "\#" were represented to significantly different with untreated control and PG/Dox alone, respectively

\section{Death property of PG/Dox-induced cell death in normal cells}

PG-priming reinforced Dox's cytotoxicity via activating autophagy [19]. Therefore, autophagy might be stimulated in normal cells by PG-priming. Moreover, cell viability slightly decreased from combining with autophagic inhibitors except for HK-2 coupling with bafilomycin A1 (Fig. 2). This result revealed the different mechanisms of PG/Dox combination and the autophagy might play a protective role in normal cells instead of cytotoxic role in OSCC [19]. Afterward, cell cycle analysis was performed to evaluate the linkage of apoptosis and PG/Dox cytotoxic enhancement in normal cells. When compared with Dox as a baseline, sub- $\mathrm{G}_{1}$ in PG/ Dox-treated FL83B and h9c2 cells was significantly raised, whereas HK-2 showed no significant differences (Fig. 3). In FL83B cells, elevated sub- $\mathrm{G}_{1}$ was contributed from decrease of $G_{0} / G_{1}$ and $G_{2} / M$. In $h 9 c 2$, increase of sub- $G_{1}$ mainly came from $\mathrm{G}_{0} / \mathrm{G}_{1}$ decrease (Fig. 3). Moreover, $\mathrm{S}$ phase in PG/ Dox-treated FL83B and h9c2 cells significantly elevated from $22.9 \pm 0.4 \%$ to $28.4 \pm 0.4 \%$ (FL83B) and $5.5 \pm 0.1 \%$ to $9.1 \pm 1.2 \%$ (h9c2), which was associated with the topoisomerase IIb inhibitory property of Dox (Fig. 3). In HK-2, it was interesting that $S$ phase decreased and $\mathrm{G}_{2} / \mathrm{M}$ phase significantly increased in PG/Dox group when compared with Dox alone group (Fig. 3). That is, cytotoxic enhancement of PG-priming in HK-2 might be achieved through a different approach that has no linkage with autophagy. Importantly, all three cell lines showed sub- $\mathrm{G}_{1}$ increases in the PG alone group and were higher than the Dox-treated group, which meant that PG might be more toxic than Dox in normal cells. Detail triggers of PG-induced Dox cytotoxic enhancement were further examined via three strategies: oxidative stress, Dox influx, and Dox metabolism.

\section{Discover the triggering mechanism of PG/Dox synergism in normal cells}

\section{Dox influx alteration after PG-priming}

In our previous study, PG-induced autophagy that increased Dox accumulation through upregulating veiled Dox importer expression has been demonstrated [19]. Accordingly, Dox influx would be the first strategy for testing the mechanism of PG-promoted Dox cytotoxicity in normal cells. In addition, only importer expressions were affected by PG-activated autophagy. Therefore, allosteric inhibition would not be determined during Dox influx analysis. Interestingly, Dox influx significantly declined in normal cells after 12-h PGpretreatment (Fig. 4). This result excluded the involvement of Dox influx in PG-promoted Dox cytotoxicity.

\section{Dox-related metabolism alteration after PG/Dox synergism}

The reduction of Dox metabolism might contribute to Dox accumulation in either OSCC cells or normal cells. The experiment determined whether PG-priming altered Dox metabolism and subsequently raised Dox accumulation. The final enzymes of three metabolic pathways in Dox metabolism-Aldo/Keto reductase (AKR1A1), NAD(P)H quinone dehydrogenase (NQO1), and NADH:Ubiquinone oxidoreductase (NDUFS1, also known as mitochondrial complex 1)—were overexpressed by lentiviral activation particle sequentially selection with hygromycin S. In welltransfected cells, only HK-2 showed cell viability increases (Fig. 5). Combining results from gene overexpression, 


\section{(A) FL83B}

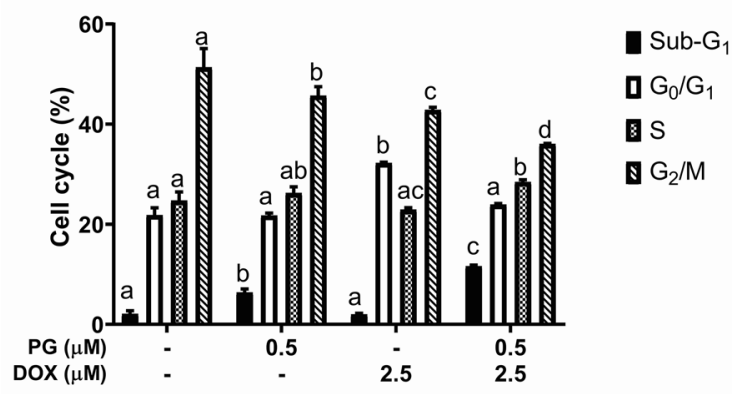

(C) h9c2

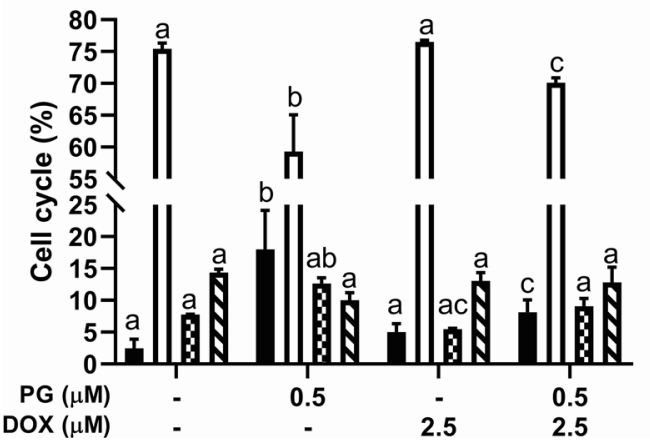

Fig. 3 Cell cycle change in normal cells after PG/Dox treatment. FL83B, HK-2, and h9c2 were treated with PG and Dox, respectively, stained with PI, and determined intracellular fluorescent intensity by flow cytometry. Data were summarized from three independent

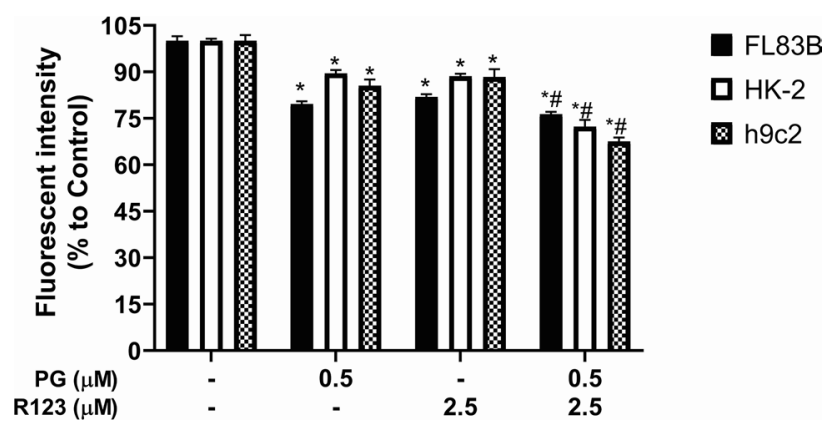

Fig. 4 Normal cells' Dox influx altered by PG-priming. Normal cells were treated with PG prior to rhodamine 123 (R123) and then intracellular fluorescent intensity was measured. Data were normalized to untreated control and shown in mean \pm SD from four independent experiments. Significant difference $(p<0.05)$ was labeled with “*” or “\#” as compared with untreated control (*) or Dox alone (\#)

metabolic reduction of Dox was comprised in PG/Dox synergism in HK-2 cells, but not in FL83B cells.

\section{Sustained cytotoxicity of PG/Dox synergism in normal cells}

For clinical applications of PG/Dox synergism, the cytotoxic change after PG/Dox treatment in normal cells was

\section{(B) HK-2}

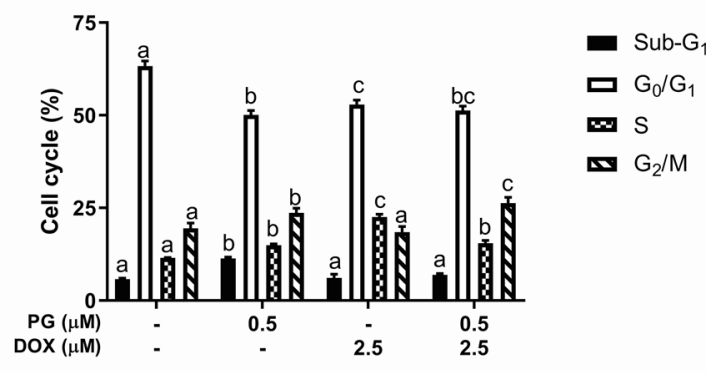

experiments and shown in mean \pm SD which were marked with "*” or "\#” as significantly different $(p<0.05)$ with untreated control or Dox alone

measured. Subsequently, tested normal cells were treated with PG/Dox, renewed fresh cultural medium, and cultured additional for 2 days to determine post-treatment cytotoxic alteration in normal cells. Unfortunately, after 2 days culture, cell viability of all cells was continuously decreased to $13.5 \pm 0.6 \%$ (FL83B), $11.1 \pm 1.1 \%$ (HK-2), and $27.4 \pm 0.4 \%$ (h9c2), respectively (Fig. 6), which meant sustained cytotoxic event was observed in normal cells and consequently could underestimate the cytotoxicity of PG/Dox synergism.

\section{Discussion}

The result of this study can be seen as the two following approaches: (1) toxicity examination of PG/Dox synergism in normal cell; and (2) metabolic interference of Dox by PG/Dox synergism. This study demonstrated that cytotoxicity of PG/Dox synergism against normal cells was less than for OSCC cells and putatively might work through different pathways. Furthermore, PG/Dox synergism reduced Dox metabolism and led to increases in Dox accumulation in normal cells. However, sustained cytotoxicity of PG/Dox synergism could be found in normal cells and the under-estimation of cytotoxic efficacy against OSCC and its potential toxicity toward normal cells. 
(A) Hygromycin S

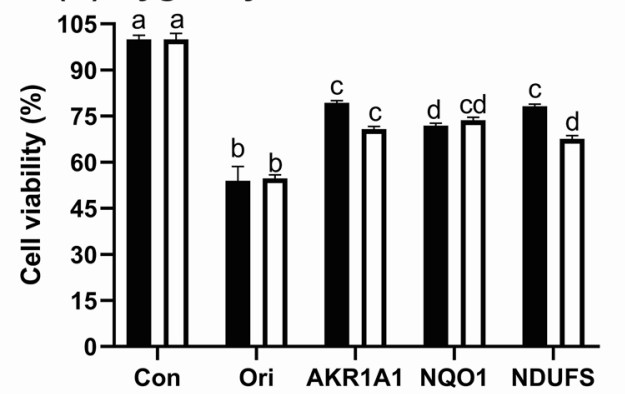

(B) PG/Dox

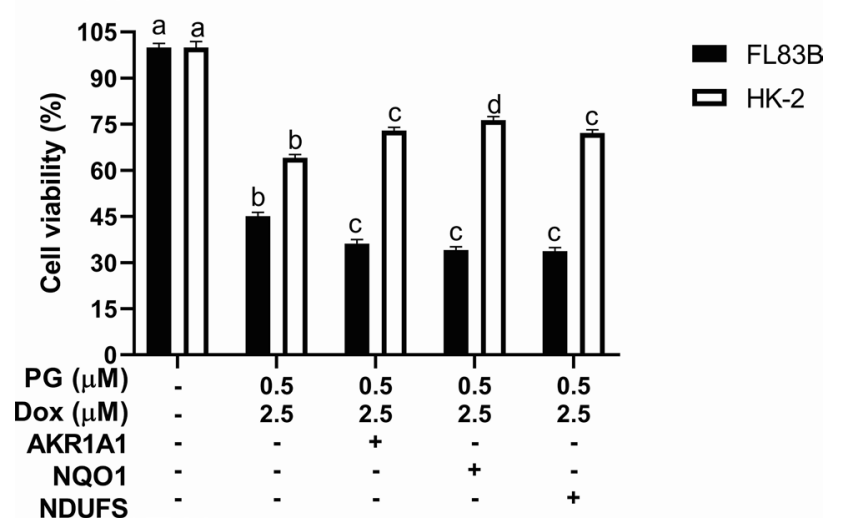

Fig. 5 Cytotoxic change of PG/Dox synergism in normal cells after gene overexpression. Normal cells were overexpressed AKR1A1 and NQO1 via lentivirus transfection and selected through hygromycin S treatment. Then, transfected cells were treated with a hygromycin $\mathrm{S}$ and $\mathbf{b}$ PG/Dox to evaluate transfection success and impact of Doxmetabolic enzyme on PG/Dox synergism. Data were represented with mean \pm SD from four independent experiments. Different letters on the columns meant significantly different to each other $(p<0.05)$

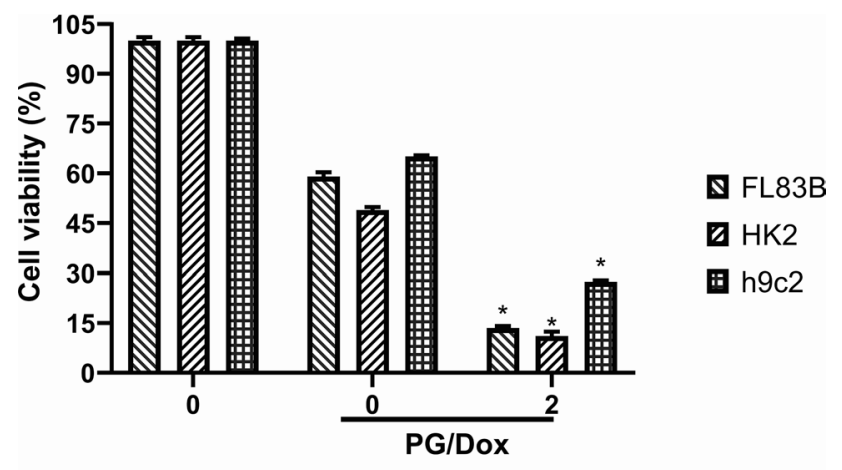

Day after treatment

Fig. 6 Sustained effect of PG/Dox in normal cells and OSCC. Normal cells were treated with PG/Dox for $12 / 12 \mathrm{~h}$ and renewed cultural medium for additional incubation for 2 days and determined cell viability by MTT assay. Results were shown in mean \pm SD from 4 independent experiments and labeled with "*" as significantly different with PG/Dox treatment at Day 0
In the past 20 years, $P G$ has been investigated in numerous biological functions with strong antitumor activity against a wide variety of cell type cancers [20]. Moreover, this study and our previous works have reported the chemosensitization of PG combined with Dox or paclitaxel for promoting the tumoricidal effect of these chemotherapeutic drugs [19, 21]. However, the pharmacodynamic (PD) and pharmacokinetic (PK) data for PG are still absent. The only information about PG's PK/PD is its $\mathrm{LD}_{50}$, which is 26-30 $\mu \mathrm{g} / \mathrm{egg}$ [22]. Chen et al. tested acute and hereditary toxicity of PG in mice, which showed the $\mathrm{LD}_{50}$ of PG in Kunming mice is larger than $10 \mathrm{~g} / \mathrm{kg}$ and no putative hereditary toxicity was observed [23]. Other PK/PD information such as tissue distribution, absorption rate, eliminating route, and clearance is absent, which leads the clinical application of PG impossible. Accordingly, filling PK/PD data of PG is critical for clinical application of PG.

Due to high incidence in Dox-mediated cardiotoxicity, numerous researches work on the formulation or compound combination that could relief Dox-mediated toxicity. For ameliorating Dox-induced toxicity, antioxidants will be the first choice in filtrating candidates according to the ROSinducing mechanism in Dox-induced toxicity and nature compounds with antioxidant activity being usually provided as a positive effect in relieving Dox-induced toxicity $[9,24]$. The application of Traditional Chinese Medicine or herbal products in attenuation of Dox-induced cardiotoxicity, Qishen Yiqi Dropping Pills, beet root juice, and Ginkgo biloba extract shows different relieving mechanisms in vivo and in vitro in which Qishen Yiqi Dropping Pills upregulates VEGF expression in myocardio tissues, and beet root juice and G. biloba extract prevent the apoptosis of cardiomyocytes through modulating $\mathrm{Bcl}-2 / \mathrm{Bax}$ ratio [25-27]. Other than herbal products, 3,3'-Diindolylmethane and eriodictyol-7-O-glucoside activated Nrf-2-related anti-oxidant response element pathway to reduce Doxand cisplatin-induced DNA damage [28, 29]. 6-Gingerol, Myricitrin, isorhamnetin, glabridin, and tanshinone IIA reduce cardiomyocyte apoptosis through activating growth factor signaling and preventing mitochondrial dysfunction [30-34]. Isodunnianol from Illicium verum activates protective autophagy through AMPK/ULK1 signaling pathway [35]. Comparing to our results, PG exacerbates Dox toxicity against oral squamous cell carcinoma cells through the augmenting of Dox influx which could not be observed in normal cell lines [19], which could not be found in identified Dox protectors. Our results elicit a new path for building distinct cytotoxicity of chemotherapy drug against cancer cell and normal cell to hinder its putative toxicity.

Another important finding of this study is the sustained cytotoxicity of PG/Dox synergism in normal cells even when the treatment came to an endpoint. From this finding evolved two concerned issues: (1) cytotoxicity of 
experimental observations needs extend to post-treatment; and (2) under-estimation of PG/Dox's cytotoxicity. Mostly, sustained cytotoxicity is applied to describe the continuous release of packaged chemicals in a specific tissue such as wogonin nanoparticles against breast cancer [36], natamycin solid lipid nanoparticles in treating fungal keratitis [37], and self-assembly herbal hydrogels for neural inflammation [38]. For evaluations of small compound toxicity, this study is the first report on the impact of post-treatment cytotoxicity, while the detail mechanism after drug removal is entirely unclear. From previous study, oxidative stress was found to be one conceivable mechanism after drug removal which is the main cause of Dox-mediated cardiotoxicity and that also could be observed in normal cells during treatments [9]. When taking post-treatment cytotoxicity into considerations, current formulation of PG and Dox is appropriate and needs more investigation (dose or period of PG and Dox treated) to optimize the tumoricidal efficacy and minimize the normal cell toxicity.

\section{Conclusion}

Of note, lowering normal cell cytotoxicity of PG/Dox synergism was reported and further reinforced the potential of PG/ Dox for clinical application. Furthermore, the identification of PG in this study as a Dox-metabolic modulator resulted in the augment of Dox accumulation via either Dox importation or Dox metabolism. For future clinical application, the most critical events are the refilling PK/PD of PG and optimizing the PG/Dox regimen with considering cytotoxicity of post-treatment.

Author contributions $\mathrm{CF}$, FS, and TJ constructed the concept of whole study, SR and CC performed investigation, CS validated the data and carried on statistical analysis, SR and LB wrote the original manuscript, FS, TJ, and CC edited and revised the manuscript, and CS supervised the project and funding support. All authors had read the manuscript and approved the publication of this manuscript.

Funding This study was supported by Hualien Armed Forces General Hospital, Hualien, Taiwan (HAFGH-D-109014).

\section{Compliance with ethical standards}

Conflict of interest The authors have claimed no competing interests in this study.

Open Access This article is licensed under a Creative Commons Attribution 4.0 International License, which permits use, sharing, adaptation, distribution and reproduction in any medium or format, as long as you give appropriate credit to the original author(s) and the source, provide a link to the Creative Commons licence, and indicate if changes were made. The images or other third party material in this article are included in the article's Creative Commons licence, unless indicated otherwise in a credit line to the material. If material is not included in the article's Creative Commons licence and your intended use is not permitted by statutory regulation or exceeds the permitted use, you will need to obtain permission directly from the copyright holder. To view a copy of this licence, visit http://creativecommons.org/licenses/by/4.0/.

\section{References}

1. Johnson-Arbor K, Dubey R (2018) Doxorubicin. StatPearls, Treasure Island

2. Ballet F, Vrignaud P, Robert J, Rey C, Poupon R (1987) Hepatic extraction, metabolism and biliary excretion of doxorubicin in the isolated perfused rat liver. Cancer Chemother Pharmacol 19:240-245. https://doi.org/10.1007/BF00252979

3. Kwok KK, Vincent EC and Gibson JN (2017) Antineoplastic drugs. In: Pharmacology and therapeutics for dentistry, pp 530-562

4. Licata S, Saponiero A, Mordente A, Minotti G (2000) Doxorubicin metabolism and toxicity in human myocardium: role of cytoplasmic deglycosidation and carbonyl reduction. Chem Res Toxicol 13:414-420. https://doi.org/10.1021/tx000013q

5. Asensio-Lopez MC, Soler F, Pascual-Figal D, Fernandez-Belda F, Lax A (2017) Doxorubicin-induced oxidative stress: the protective effect of nicorandil on HL-1 cardiomyocytes. PLoS ONE 12:e0172803. https://doi.org/10.1371/journal.pone.0172803

6. Damiani RM, Moura DJ, Viau CM, Caceres RA, Henriques JAP, Saffi J (2016) Pathways of cardiac toxicity: comparison between chemotherapeutic drugs doxorubicin and mitoxantrone. Arch Toxicol 90:2063-2076. https://doi.org/10.1007/s00204-016-1759-y

7. Renu K, Abilash VG, Tirupathi Pichiah PB, Arunachalam S (2018) Molecular mechanism of doxorubicin-induced cardiomyopathy—an update. Eur J Pharmacol 818:241-253. https://doi. org/10.1016/j.ejphar.2017.10.043

8. Baxter-Holland M, Dass CR (2018) Doxorubicin, mesenchymal stem cell toxicity and antitumour activity: implications for clinical use. J Pharm Pharmacol 70:320-327. https://doi.org/10.1111/ jphp. 12869

9. Pugazhendhi A, Edison T, Velmurugan BK, Jacob JA, Karuppusamy I (2018) Toxicity of Doxorubicin (Dox) to different experimental organ systems. Life Sci 200:26-30. https://doi. org/10.1016/j.lfs.2018.03.023

10. Chatterjee K, Zhang J, Honbo N, Karliner JS (2010) Doxorubicin cardiomyopathy. Cardiology 115:155-162. https://doi. org/10.1159/000265166

11. Kaminskas LM, McLeod VM, Kelly BD, Sberna G, Boyd BJ, Williamson M, Owen DJ, Porter CJ (2012) A comparison of changes to doxorubicin pharmacokinetics, antitumor activity, and toxicity mediated by PEGylated dendrimer and PEGylated liposome drug delivery systems. Nanomedicine 8:103-111. https://doi. org/10.1016/j.nano.2011.05.013

12. Fessele KL (2017) Financial toxicity: management as an adverse effect of cancer treatment. Clin J Oncol Nurs 21:762-764. https ://doi.org/10.1188/17.CJON.762-764

13. Zhang A, Sun H, Wang X (2014) Potentiating therapeutic effects by enhancing synergism based on active constituents from traditional medicine. Phytother Res 28:526-533. https://doi. org/10.1002/ptr.5032

14. National Cancer Institute (2002) Breast cancer treatment (adult) $(\mathrm{PDQ}(\mathrm{R}))$ : health professional version. PDQ Cancer Information Summaries, Bethesda

15. Formica V, Zaniboni A, Loupakis F, Roselli M (2018) Noninferiority of three months versus six months of oxaliplatin-based 
adjuvant chemotherapy for resected colon cancer. How should IDEA findings affect clinical practice? Int J Cancer 143:23422350. https://doi.org/10.1002/ijc.31616

16. Wezel F, Vallo S, Roghmann F, Young Academic Urologist Urothelial Carcinoma Group of the European Association of $U$ (2017) Do we have biomarkers to predict response to neoadjuvant and adjuvant chemotherapy and immunotherapy in bladder cancer? Transl Androl Urol 6:1067-1080. https://doi.org/10.21037/ tau.2017.09.18

17. Agarwala V, Choudhary N, Gupta S (2017) A risk-benefit assessment approach to selection of adjuvant chemotherapy in elderly patients with early breast cancer: a mini review. Indian J Med Paediatr Oncol 38:526-534. https://doi.org/10.4103/ijmpo.ijmpo - $96 \_17$

18. Carvalho C, Glynne-Jones R (2017) Challenges behind proving efficacy of adjuvant chemotherapy after preoperative chemoradiation for rectal cancer. Lancet Oncol 18:e354-e363. https://doi. org/10.1016/S1470-2045(17)30346-7

19. Lin SR, Weng CF (2018) PG-priming enhances doxorubicin influx to trigger necrotic and autophagic cell death in oral squamous cell carcinoma. J Clin Med 7:375. https://doi.org/10.3390/jcm7100375

20. Darshan N, Manonmani HK (2015) Prodigiosin and its potential applications. J Food Sci Technol 52:5393-5407. https://doi. org/10.1007/s13197-015-1740-4

21. Ho TF, Peng YT, Chuang SM, Lin SC, Feng BL, Lu CH, Yu WJ, Chang JS, Chang CC (2009) Prodigiosin down-regulates survivin to facilitate paclitaxel sensitization in human breast carcinoma cell lines. Toxicol Appl Pharmacol 235:253-260. https://doi. org/10.1016/j.taap.2008.12.009

22. Kalesperis GS, Prahlad KV, Lynch DL (1975) Toxigenic studies with the antibiotic pigments from Serratia marcescens. Can J Microbiol 21:213-220. https://doi.org/10.1139/m75-030

23. Chen XY, Yang PZ, Jiang ST, Zheng G, Cao LL, Cao XM, Zhu XX, Zhang DF, Liu GQ (2017) Experimental study of acute oral toxicity and genetic toxicity of natural red pigment prodigiosin. Food Sci 38:224-228. https://doi.org/10.7506/spkx1002-6630201713037

24. Rejhova A, Opattova A, Cumova A, Sliva D, Vodicka P (2018) Natural compounds and combination therapy in colorectal cancer treatment. Eur J Med Chem 144:582-594. https://doi. org/10.1016/j.ejmech.2017.12.039

25. Wang LJ, Wang LY, Zhou XX, Ruan GR, Yang GY (2019) Qishen Yiqi dropping pills ameliorates doxorubicin-induced cardiotoxicity in mice via enhancement of cardiac angiogenesis. Med Sci Monit 25:2435-2444. https://doi.org/10.12659/Msm.915194

26. Das S, Filippone SM, Williams DS, Das A, Kukreja RC (2016) Beet root juice protects against doxorubicin toxicity in cardiomyocytes while enhancing apoptosis in breast cancer cells. Mol Cell Biochem 421:89-101. https://doi.org/10.1007/s11010-016-2789-8

27. Yeh YC, Liu TJ, Wang LC, Lee HW, Ting CT, Lee WL, Hung CJ, Wang KY, Lai HC, Lai HC (2009) A standardized extract of Ginkgo biloba suppresses doxorubicin-induced oxidative stress and p53-mediated mitochondrial apoptosis in rat testes. Br J Pharmacol 156:48-61. https://doi.org/10.111 1/j.1476-5381.2008.00042.x

28. Hajra S, Basu A, Singha Roy S, Patra AR, Bhattacharya S (2017) Attenuation of doxorubicin-induced cardiotoxicity and genotoxicity by an indole-based natural compound 3,3'-diindolylmethane (DIM) through activation of Nrf2/ARE signaling pathways and inhibiting apoptosis. Free Radic Res 51:812-827. https://doi.org/10.1080/10715762.2017.1381694

29. Hu Q, Zhang DD, Wang L, Lou H, Ren D (2012) Eriodictyol-7-Oglucoside, a novel Nrf2 activator, confers protection against cisplatin-induced toxicity. Food Chem Toxicol 50:1927-1932. https ://doi.org/10.1016/j.fct.2012.03.059

30. Modarresi M, Hajialyani M, Moasefi N, Ahmadi F, Hosseinzadeh L (2019) Evaluation of the cytotoxic and apoptogenic effects of glabridin and its effect on cytotoxicity and apoptosis induced by doxorubicin toward cancerous cells. Adv Pharm Bull 9:481-489. https://doi.org/10.15171/apb.2019.057

31. Wen J, Wang J, Li P, Wang R, Wang J, Zhou X, Zhang L, Li H, Wei S, Cai H, Zhao Y (2019) Protective effects of higenamine combined with [6]-gingerol against doxorubicin-induced mitochondrial dysfunction and toxicity in $\mathrm{H} 9 \mathrm{c} 2$ cells and potential mechanisms. Biomed Pharmacother 115:108881. https://doi. org/10.1016/j.biopha.2019.108881

32. Sun J, Sun GB, Cui XL, Meng XB, Qin M, Sun XB (2016) Myricitrin protects against doxorubicin-induced cardiotoxicity by counteracting oxidative stress and inhibiting mitochondrial apoptosis via ERK/P53 pathway. Evid Based Complement Altern Med 2016:6093783. https://doi.org/10.1155/2016/6093783

33. Sun J, Sun GB, Meng XB, Wang HW, Luo Y, Qin M, Ma B, Wang M, Cai DY, Guo P, Sun XB (2013) Isorhamnetin protects against doxorubicin-induced cardiotoxicity in vivo and in vitro. PLoS ONE 8:e64526. https://doi.org/10.1371/journal.pone.0064526

34. Hong HJ, Liu JC, Chen PY, Chen JJ, Chan P, Cheng TH (2012) Tanshinone IIA prevents doxorubicin-induced cardiomyocyte apoptosis through Akt-dependent pathway. Int J Cardiol 157:174179. https://doi.org/10.1016/j.ijcard.2010.12.012

35. Chen C, Jiang L, Zhang M, Pan XL, Peng C, Huang W, Jiang QL (2019) Isodunnianol alleviates doxorubicin-induced myocardial injury by activating protective autophagy. Food Funct 10:26512657. https://doi.org/10.1039/c9fo00063a

36. Baek JS, Na YG, Cho CW (2018) Sustained cytotoxicity of wogonin on breast cancer cells by encapsulation in solid lipid nanoparticles. Nanomaterials 8:159. https://doi.org/10.3390/ nano8030159

37. Khames A, Khaleel MA, El-Badawy MF, El-Nezhawy AOH (2019) Natamycin solid lipid nanoparticles-sustained ocular delivery system of higher corneal penetration against deep fungal keratitis: preparation and optimization. Int J Nanomed 14:25152531. https://doi.org/10.2147/IJN.S190502

38. Zheng J, Fan R, Wu H, Yao H, Yan Y, Liu J, Ran L, Sun Z, Yi L, Dang L, Gan P, Zheng P, Yang T, Zhang Y, Tang T, Wang Y (2019) Directed self-assembly of herbal small molecules into sustained release hydrogels for treating neural inflammation. Nat Commun 10:1604. https://doi.org/10.1038/s41467-019-09601-3

Publisher's Note Springer Nature remains neutral with regard to jurisdictional claims in published maps and institutional affiliations. 